\title{
Effect of the elastomer stiffness and coupling agents on rheological properties of magnetorheological elastomers
}

\author{
A. Boczkowska \& S. F. Awietjan \\ Faculty of Materials Science and Engineering, \\ Warsaw University of Technology, Poland
}

\begin{abstract}
The reported studies are related to a new group of intelligent materials, such as magnetorheological elastomers (MREs), which are composites of ferromagnetic particles embedded in elastomer matrix. Studies on fabrication of MREs were carried out using two different polyurethane elastomers as a matrix, ferromagnetic particles and coupling agent. The matrices were differed in elastomer stiffness and hardness. As a ferromagnetic component carbonyl-iron powder with particle size of 6-9 $\mu \mathrm{m}$ was used. Particles were oriented into chains under the external magnetic field of $240 \mathrm{kA} / \mathrm{m}$. Samples with anisotropic particles arrangement and particles volume fraction equal to $11.5 \%$ were examined. Microstructure of MREs was observed using Scanning Electron Microscopy. Structural and magnetic anisotropy of the MREs was derived from the magnetic studies. Rheological properties of the MREs, such as storage and loss modulus and loss factor, were characterized as a function of shear frequency and strength of the magnetic field. Absolute and relative magnetorheological effects were calculated taking the microstructure into account, which was formed in the course of the MRE fabrication, by changing the elastomer type and application of the coupling agent. As a result of the studies it was found that the MREs with stiffer matrix exhibit much lower MR effect than the soft ones. Also the application of coupling agent lead to a decrease of MR effect by the formation of bound elastomer surrounding the particles, which increases the stiffness of the matrix.

Keywords: magnetorheological elastomers, polyurethanes, carbonyl iron, rheological properties, coupling agents, microstructure, magnetic properties, magnetorheological effect.
\end{abstract}




\section{Introduction}

Magnetorheological elastomers (MREs) are composites of ferromagnetic particles embedded in elastomer matrix. They belong to a new group of intelligent materials, which change their rheological properties under the influence of an applied magnetic field. They are solid analogues of the magnetorheological fluids (MRF) where the matrix is a solid elastic polymer rather than carrier oil. These both consist of micrometer-sized magnetically permeable particles in a non-magnetic matrix material. As in the case of MRF, the particles try to arrange themselves in the direction of the magnetic field. An external magnetic field is applied during the curing process of the elastomer, what is described in many papers, e.g. Zhou [1], Farshad and Benine [2], Jolly et al. [3]. The field induces dipole moments within the particles, which seek minimum energy states. Chains of particles with collinear dipole moments are formed and curing of the polymeric matrix locks the chains in place. As a result special structures, such as chains or columns of particles, remain in the matrix. MREs have controllable magnetic field dependent modulus rather than field dependent yield stress and also stable performance. The advantage of MREs, in comparison to MRF, is that ferromagnetic particles do not undergo the sedimentation. Moreover, the amount of filler is usually lower in MREs. As a result, the weight of sensing and actuating devices based on MREs is reduced and due to their microstructure, the time, following signal introduction, for a strain change response to applying a magnetic field is shortened, as it was described by Carlson and Jolly [4]. MREs exhibit reversible changes of their properties and shape under the magnetic field, what makes them attractive for applications as dampers, sensors or actuators, e.g. An and Shaw [5], Farshad and Le Roux [6]. The automotive bushing based on MREs, adjusted to reduce suspension deflection and to improve passenger comfort, were patented by Watson [7] and Steward et al. [8] for Ford Motor Company.

The magnetic particles are usually carbonyl iron and as the matrix soft silicone elastomers, poly(vinyl alcohol), gelatine, natural rubber or polyurethanes can be used. The results of the studies presented in the literature [9-12] show that the properties of MREs depend on the particles shape, size and volume fraction, as well as the magnetic field strength. Only a few papers are focused on the influence of the polymer matrix properties on the magnetorheological effect (MR effect) in MREs. Gong et al. [13] and Lokander and Stenberg [9] reported the advantageously influence on the MR effect of the addition of plasticizers to the silicon rubber due to the decrease of the polymer matrix stiffness. Jiang et al. [14] indicate on the positive effect of the particles surface modification on the increase of the MR effect, while Zhang et al. [15], Fan et al. [16] and Li et al. [17] show that the enhancement of the particle-polymer matrix interaction leads to the decrease of MR effect due to the existence of bound-rubber phenomenon. The improvement of filler-matrix interaction is an effective way to improve the mechanical properties of materials; however in the case of MREs it can affect the MR effect. 
In our studies the influence of the matrix and the interaction between matrix and filler on the MREs properties were investigated. We used two polyurethane elastomers as a matrix, which were differed in stiffness and hardness. Particles were oriented into chains under the external magnetic field of $240 \mathrm{kA} / \mathrm{m}$. Samples with the same particles volume fraction equal to $11.5 \%$, were examined. The iron particles-polyurethane matrix interactions were changed by the application of silane coupling agent and the effect of the interaction on the performance of MREs were investigated. Rheological properties of the MREs, such as storage and loss modules and loss factor, were characterized as a function of shear frequency and strength of the magnetic field. Absolute and relative MR effects were calculated taking microstructure into account, which was formed in the course the MREs fabrication, by changing the elastomer type and application of the coupling agent.

\section{Materials and methods}

Magnetorheological elastomers were manufactured using:

- $\quad$ soft polyurethane (PU), obtained from polyether polyols VORALUX ${ }^{\circledR}$ HF 505 used in a blend with 14922, with the average molecular weight respectively 3596 and $4350 \mathrm{~g} / \mathrm{mol}$, and isocyanate compound HB 6013, supplied by Dow Chemical Company,

- segmented urea-urethane elastomer (EPU), obtained from 4,4'diphenylmethane diisocyanate (MDI), ethylene oligoadipate (OAE) with the average molecular weight about $2000 \mathrm{~g} / \mathrm{mol}$ and dicyandiamide (DCDA) as a chain extender.

PU substrates were mixed in the weight ratio, respectively 30:70:23. Mixing and curing process were conducted at room temperature.

EPU with molar ratio of MDI:(OAE+DCDA) equal to 2.5 was synthesized by one-shot method. The curing process was carried out at temperature of $150^{\circ} \mathrm{C}$, what makes technology aspects of MRE manufacturing more complicated. The existence in every short hard segment of strong polar urea group and strong polar nitrilimide side-group increases the urea-urethane thermal and mechanical properties, as well as stiffness and hardness in comparison to soft polyether polyurethane (see Table 1).

The ferromagnetic component used in the MREs, was carbonyl-iron powder with particles size ranging from $6-9 \mu \mathrm{m}$, produced by Fluka. The amount of the carbonyl-iron particles was equal to $11.5 \mathrm{vol} \%$. It was found in our earlier studies, e.g. Boczkowska and Awietjan [18], carried out for the series of samples with the particles content varied from 1.5 to 33 vol.\%, that the highest structural and magnetic anisotropy, as well as MR effect was observed for MREs containing 11.5 vol.\% of carbonyl-iron. Therefore, in this study such volume fraction of particles was used.

As shown in Table 1 polyurethane PU 70/30 is characterized by lower density and mechanical properties than EPU 2.5. Low hardness and stiffness of the polyurethane matrix can lead to the higher relative property changes of the MRE under an external magnetic field. On the other hand, the EPU 2.5 is distinguished 
by lower viscosity of reactive mixture of substrates and good mechanical properties. Low viscosity during the processing of the MRE makes the arrangement of the particles into aligned chains very easy.

Table 1: $\quad$ Selected physical and mechanical properties of elastomers used for MREs fabrication.

\begin{tabular}{|c|c|c|c|c|c|c|}
\hline Material & \multirow{2}{*}{$\begin{array}{c}\text { Viscosity } \\
(\mathrm{mPas})\end{array}$} & \multirow{2}{*}{$\begin{array}{c}\text { Density } \\
\left(\mathrm{g} / \mathrm{cm}^{3}\right)\end{array}$} & \multicolumn{2}{|c|}{$\begin{array}{c}\text { Glass transition } \\
\text { temperature }\left({ }^{\circ} \mathrm{C}\right)\end{array}$} & \multirow{2}{*}{$\begin{array}{c}\text { Hardness } \\
\left({ }^{\circ} \mathrm{ShA}\right)\end{array}$} & $\begin{array}{c}\text { Young's } \\
\text { modulus } \\
(\mathrm{MPa})\end{array}$ \\
\cline { 4 - 5 } & & & $\begin{array}{c}\text { Soft } \\
\text { segments }\end{array}$ & $\begin{array}{c}\text { Hard } \\
\text { segments }\end{array}$ & & \\
\hline PU 70/30 & 8000 & 1.03 & -64 & 41 & $<10$ & 0.1 \\
\hline EPU 2.5 & 1400 & 1.26 & -23 & 165 & 87 & 14 \\
\hline
\end{tabular}

The samples were subjected to a magnetic field during curing to produce aligned carbonyl-iron chains within the elastomer. The magnetic field strength used during MREs processing was equal to $240 \mathrm{kA} / \mathrm{m}$. Samples prepared for rheological measurements were in the form of $2 \mathrm{~mm}$ thick and $20 \mathrm{~mm}$ in diameter round plates.

As a coupling agent N-2-(aminoethyl)-3-aminopropyltrimethoxysilane (U-15) was used with the chemical formula $\mathrm{H}_{2} \mathrm{NCH}_{2} \mathrm{CH}_{2} \mathrm{CH}_{2} \mathrm{Si}\left(\mathrm{OC}_{2} \mathrm{H}_{5}\right)_{3}$, supplied by Unisil Tarnow Company. Carbonyl-iron particles were mechanically stirred in the $1 \%$ solution of coupling agent in toluene before MREs fabrication, until the solvent evaporation.

The microstructure of cross-sections of MREs was observed using scanning electron microscopy (SEM) Hitachi S-2600. The smooth surfaces were cut on Rotary Microtome LEICA RM2165 with LN21 cooling device.

Magnetic properties were studied by Lake Shore vibrating sample magnetometer (VSM). Tests were carried out parallel and perpendicular to the direction of particles alignment, corresponding to the magnetic field direction during curing.

Rheological properties were evaluated at $25^{\circ} \mathrm{C}$ with the application of Ares Rheometer from TA Instruments with plate-plate setup (plate diameter - $20 \mathrm{~mm}$, gap - $2 \mathrm{~mm}$, magnetic field ranged from 0 to $480 \mathrm{kA} / \mathrm{m}$ ). The chains of particles were aligned parallel to the magnetic field direction during experiment in the case of investigation of the influence of the matrix properties or they were sloped to 45 degrees in the case of the examination of the influence of coupling agent.

\section{Results and discussion}

\subsection{Microstructure observations}

Microstructure observations by SEM proved the existence of aligned particles chains in the direction of the magnetic field applied during curing of the matrix in PU 70/30 polyurethane soft elastomer (fig. 1a), as well as in EPU 2.5 ureaurethane elastomer (fig. 1b). The direction of the magnetic field during curing is shown by the white arrows in figs. $1 \mathrm{a}, 1 \mathrm{~b}$. 
a)

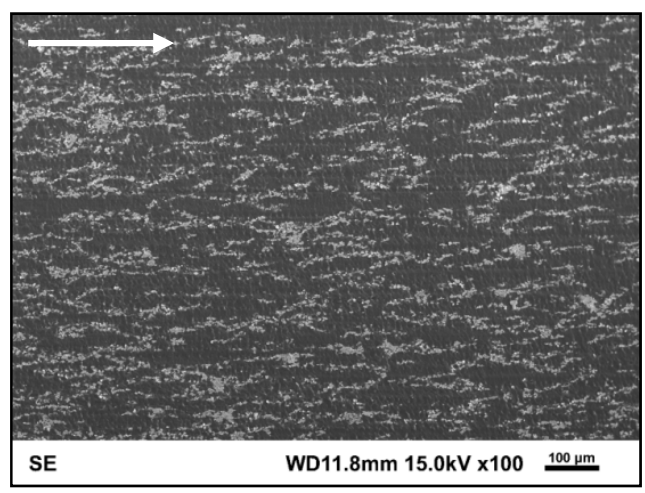

b)

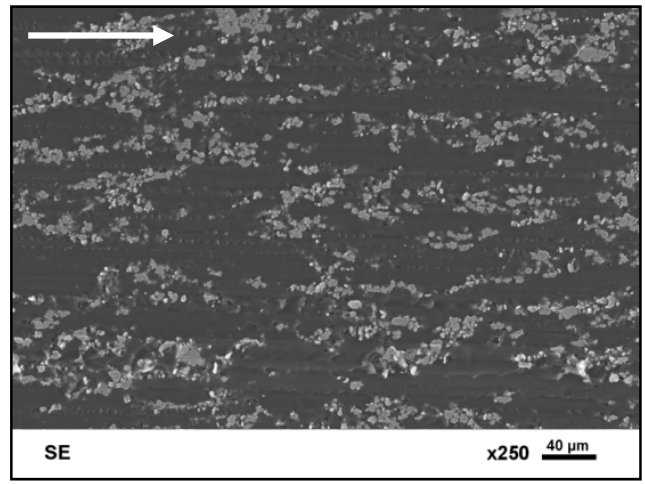

Figure 1: $\quad$ SEM images of the cross sections of MREs with 11.5 vol.\% of particles, obtained from elastomers: a) PU 70/30 polyurethane, b) EPU 2.5 poly(urea-urethane).

Although the viscosity and the chemical composition of the reactive mixtures of substrates are different, the alignment of particles into chains in both cases was obtained. The average distance among the chains of particles calculated using image analysis, as described in Boczkowska et al. [19], is equal to $40 \pm 4$ $\mu \mathrm{m}$.

\subsection{Magnetic measurements}

The existence of structural and magnetic anisotropy was confirmed by VSM studies. Tests were carried out parallel (II) and perpendicular (L) to the direction of particles chains, corresponding to the magnetic field direction during curing. 
a)

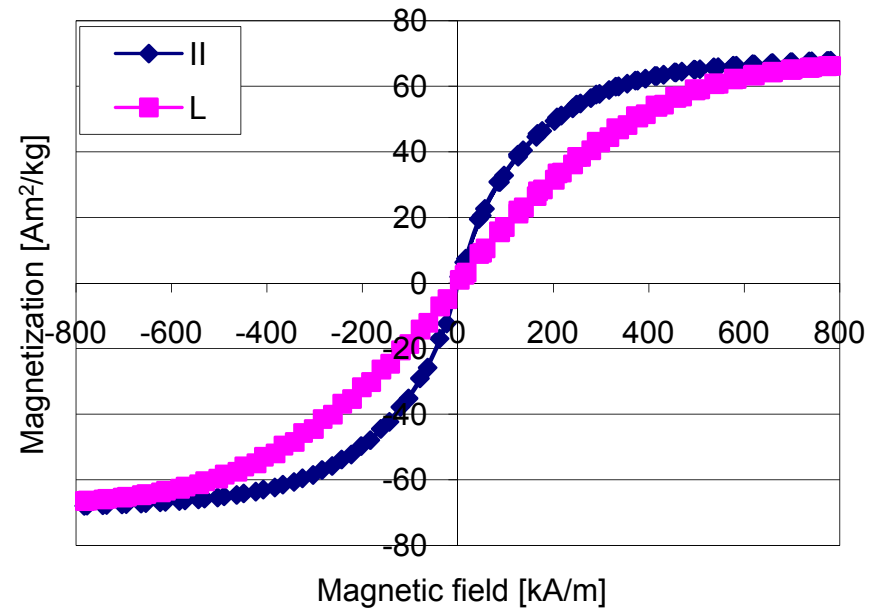

b)

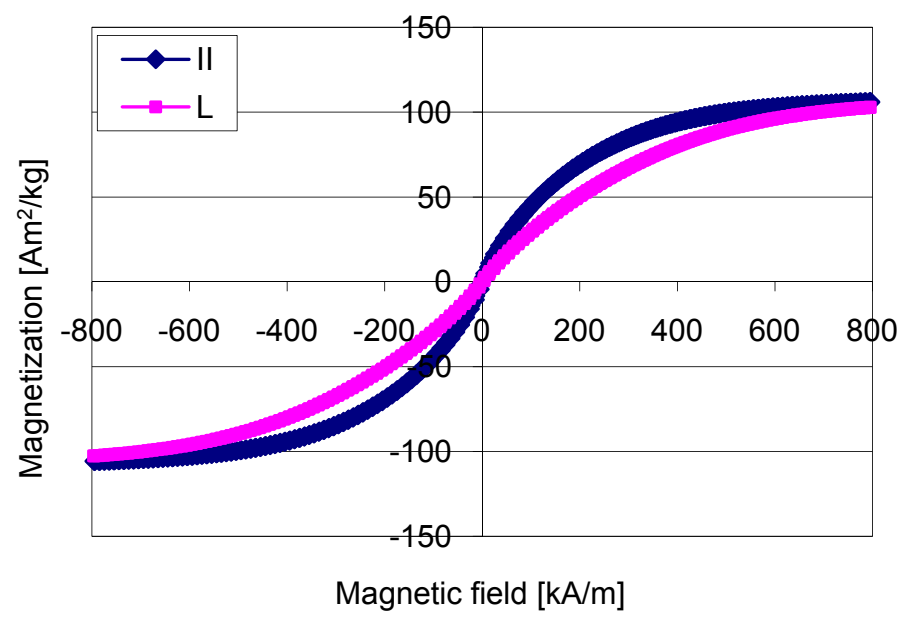

Figure 2: $\quad$ Hysteresis loops for MREs with 11.5 vol.\% of particles obtained from elastomers: a) PU 70/30 polyurethane, b) EPU 2.5 poly(ureaurethane).

In fig. 2 hysteresis loops obtained for the MREs with different matrix material are shown. From the hysteresis loops the anisotropy coefficient $\left(A_{b}\right)$ was calculated at the selected value of magnetic field strength of $160 \mathrm{kA} / \mathrm{m}$. It is expressed by the ratio of magnetization measured at $160 \mathrm{kA} / \mathrm{m}$, respectively parallel and perpendicular to the particles alignment direction. The $\mathrm{A}_{\mathrm{b}}$ values are similar for both kind of MREs matrix. They are equal to 1.50 for PU 70/30 and 1.42 for EPU 2.5, what means that the difference in viscosity and the chemical 
composition of the reactive mixtures of substrates for both elastomers do not significantly influence on the possibility of particles to arrange themselves along the magnetic field direction.

\subsection{Rheological properties}

In this study such rheological properties as storage modulus ( $\left.\mathrm{G}^{\prime}\right)$, loss modulus $\left(G^{\prime \prime}\right)$ and a ratio of G' to G', so called loss angle, were measured as a function of oscillation frequency and the magnetic field strength.

In viscoelastic materials, some of the deformation energy is stored and recovered during each cycle and some is dissipated as heat. The storage modulus represents the capacity of the material to store the energy of deformation, which contributes to the material stiffness. The loss modulus represents the capacity of the material to dissipate the energy of deformation into heat. Loss angle is a measure of material damping properties.

Rheological studies were carried out for the MRE samples fabricated using PU 70/30 elastomer without and with U-15 coupling agent. Also the influence of the matrix hardness and stiffness on rheological properties was investigated for PU 70/30 and EPU 2.5 elastomers. The examples of G', G', and tan $\delta$ curves for the MREs based on PU 70/30, obtained without and with coupling agent, as a function of the oscillation frequency, for different magnetic field strengths are shown in fig. 3.

Storage and loss modulus grow as a function of oscillation frequency. The values of G' and G' increase with the increase of the magnetic field strength. The values of G' and G" for MREs without coupling agent measured without magnetic field exhibit the lowest course, what means that such samples are characterized by lower stiffness than the samples with coupling agent.

The improvement of the adhesion between iron particles and polyurethane matrix leads to the immobilizing of the macromolecules around the particles, what increases the stiffness of the polyurethane in the area surrounding the particles. On the other hand MREs with coupling agent exhibit higher values of loss angle what is a measure of damping properties. The higher values of $\tan \delta$ mean that the better are the damping properties of such material. Loss angle increases with the increase of the magnetic field strength, and the highest values were obtained for MREs with coupling agent. However, significantly higher growth in $G^{\prime}$ and $G$ " under the magnetic field was observed for the samples without coupling agent.

Absolute $\left(\Delta \mathrm{G}^{\prime}\right)$ and relative $\left(\Delta \mathrm{G}^{\prime} / \mathrm{G}^{\prime}{ }_{0}\right)$ magnetorheological effects were calculated from rheological curves shown in fig. 3, for the same selected frequency equal to $10 \mathrm{~Hz}$. The results have been presented in fig. 4 as a function of the magnetic field strength, where $\mathrm{G}_{0}$ is the "zero field" modulus that means the storage modulus obtained without magnetic field. 

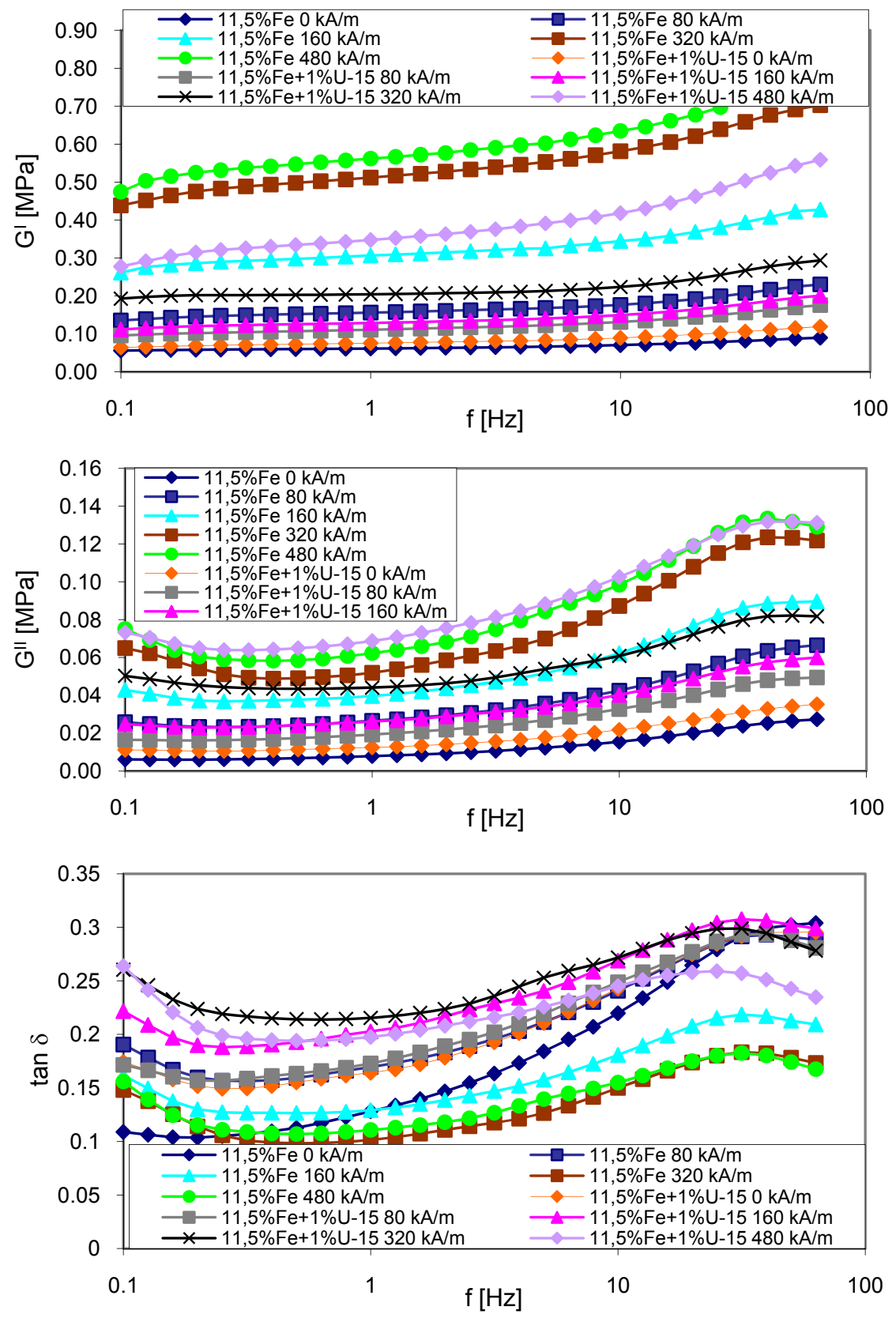

Figure 3: $\quad$ Changes of storage (G'), loss (G') modulus and loss angle ( $\tan \delta$ ) as a function of frequency under magnetic field of $0,80,160,320$, $480 \mathrm{kA} / \mathrm{m}$. 

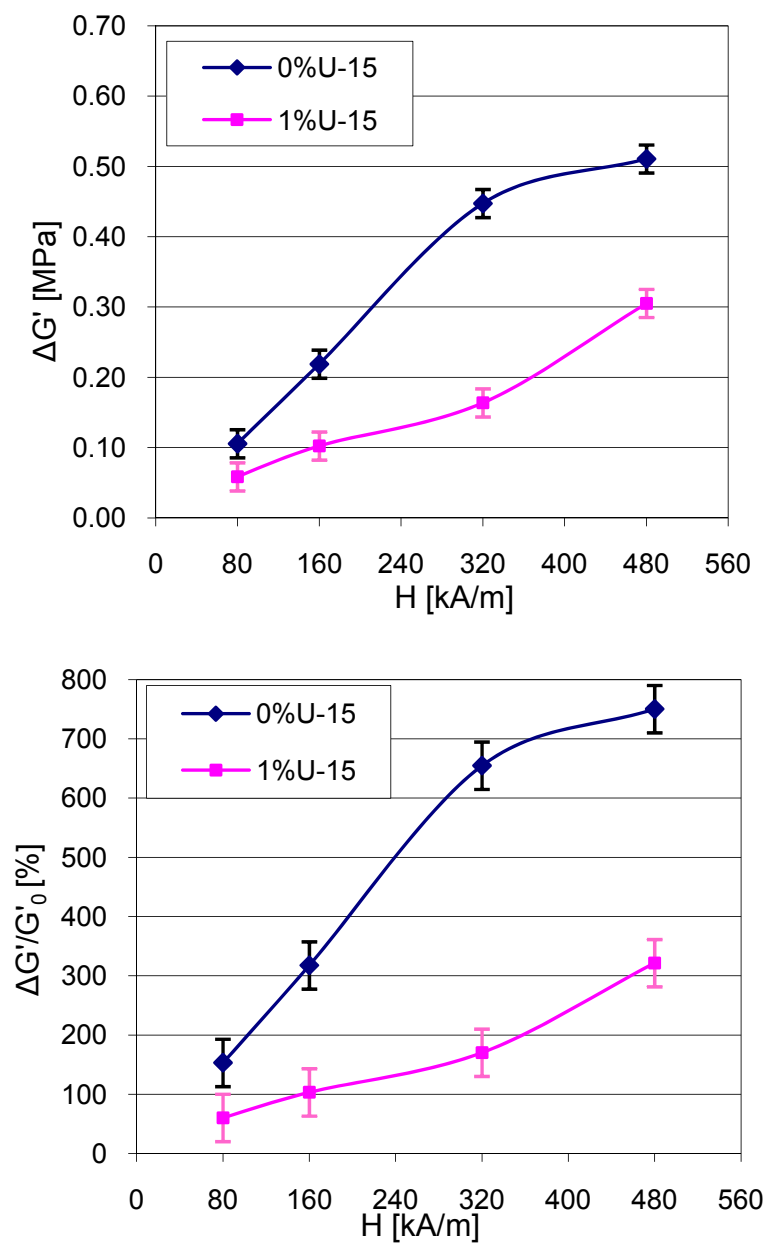

Figure 4: Absolute $\left(\Delta \mathrm{G}^{\prime}\right)$ and relative $\left(\Delta \mathrm{G}^{\prime} / \mathrm{G}^{\prime}{ }_{0}\right)$ MR effects of the MREs based on PU 70/30 obtained without and with the silane coupling agent vs. the magnetic field strength $(\mathrm{H})$.

Both MR effects, absolute and relative, grow with the magnetic field growth. The significant difference is observed between samples fabricated with and without application of silane coupling agent. The difference is much more significant with the magnetic field growth. The MR effect is higher in the MREs obtained without the application of the coupling agent because of the lower stiffness of the polyurethane matrix, what makes the ferromagnetic particles easier to attract under the magnetic field and as a result to introduce the additional strain and stress to the polyurethane matrix.

Similar results were obtained for MREs fabricated from elastomers with different stiffness and hardness, as shown in fig. 6. 

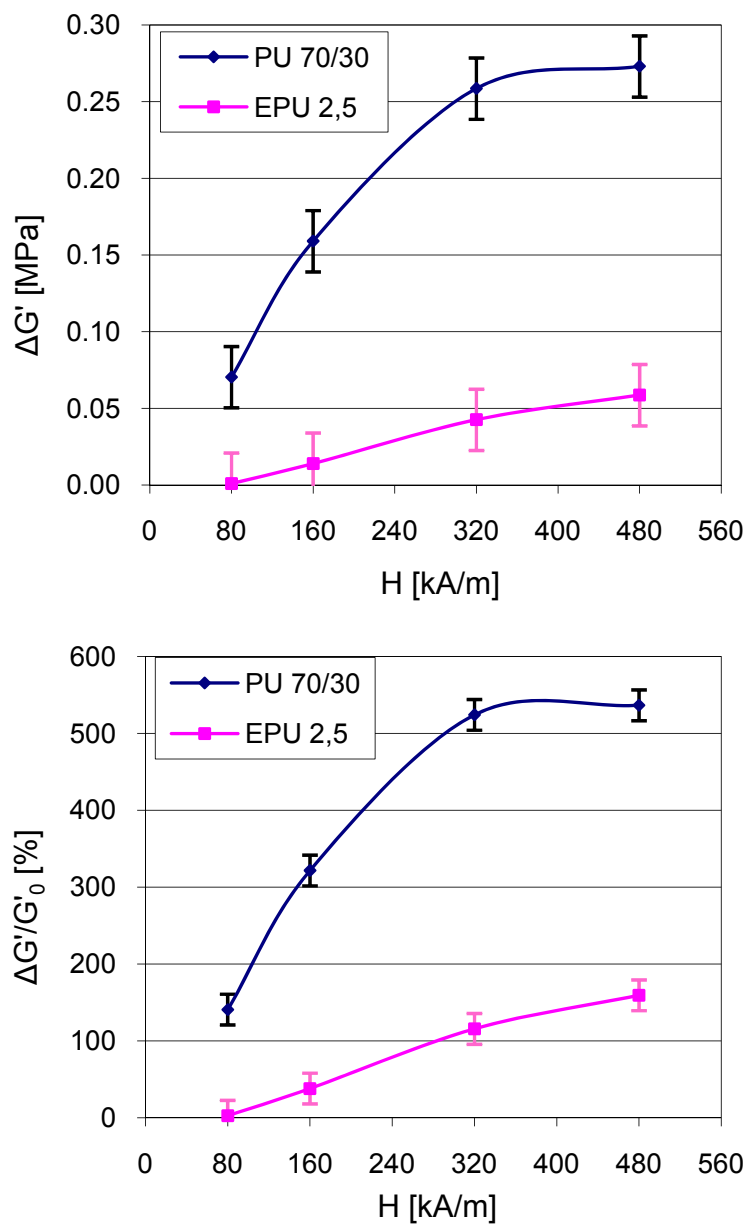

Figure 5: Absolute and relative MR effect of the MREs based on PU 70/30 and EPU 2.5 elastomers vs. the magnetic field strength $(\mathrm{H})$.

MREs with the stiffer matrix (EPU 2.5) exhibit significant lower MR effect because the stiffer matrix makes impossible the particles to displace when they are subjected to the magnetic field.

\section{Conclusions}

The properties of the MREs elastomeric matrix have a great influence on their rheological properties. Elastomer with higher stiffness and hardness leads to the higher storage and loss modulus of the MREs measured without magnetic field, while under the magnetic field the values are significant lower in comparison to 
MREs obtained from soft elastomer. As a result the absolute and relative MR effect is much higher in MREs obtained from soft elastomer.

The highest absolute and relative MR effect was obtained for the MREs synthesized from PU 70/30 elastomer with 11.5 vol.\% of carbonyl-iron particles. The values of MR effects under the magnetic field of $480 \mathrm{kA} / \mathrm{m}$ reached 0.27 $\mathrm{MPa}$ and $540 \%$, respectively, when the particles chains were aligned parallel to the magnetic field direction or $0.52 \mathrm{MPa}$ and $750 \%$, when the chains of particles were sloped to 45 degrees. The influence of the particles chains arrangement on the rheological properties is the subject of our different study.

The increasing of the adhesion between particles and polyurethane matrix leads to the significant decrease in the MR effect by the existence of bound elastomer around the particles, what makes the matrix stiffer. High stiffness of the MREs matrix do not allow for the particles displacement under the magnetic field and subsequently the deformation of the elastomeric matrix.

The rheological properties of MREs depend also on the oscillation frequency and magnetic field strength.

\section{Acknowledgement}

This work was financed by National Centre for Research and Development (Poland) as a grant no. NR 15001004.

\section{References}

[1] Zhou G.Y., Shear properties of magnetorheological elastomer, Smart Materials and Structures, 12, pp. 139-146, 2003.

[2] Farshad M. and Benine A., Magnetoactive elastomer composites, Polymer Testing, 23, pp. 347-353, 2004.

[3] Jolly M. R., Carlson J.D., Munoz B.C. and Bullions T.A., The Magnetoviscoelastic Response of Elastomer Composite Consisting of Ferrous Particles Embedded in a Polymer Matrix, Journal of Intelligent Material Systems and Structures, 7, pp. 613-622, 1996.

[4] Carlson J. D., Jolly M. R., MR fluid, foam and elastomer devices, Mechatronics, 10, pp. 555-569, 2000.

[5] An Y., Shaw M.T., Actuating properties of soft gels with ordered iron particles: basis for a shear actuator, Smart Materials and Structures, 12, pp.157-163, 2003.

[6] Farshad M., Le Roux M., Compression properties of magnetostrictive polymer composite gels, Polymer Testing, 24, pp.163-168, 2005.

[7] Watson J. R., Methods and apparatus for varying the stiffness of a suspension bushing, US Patent 5.609.353, 1997.

[8] Stewart W. M., Ginder J. M., Elie L. D., Nicholls M. E., Method and apparatus for reducing brake shudder, US Patent 5.816.587, 1998.

[9] Lokander M., Stenberg B., Improving the magnetorheological effect in isotropic magnetorheological rubber materials, Polymer Testing, 22, pp. 677-680, 2003. 
[10] Lokander M., Stenberg B., Performance of isotropic magnetorheological rubber materials, Polymer Testing, 22, pp. 245-251, 2003.

[11] Zhou, G.Y., Jiang, Z.J., Deformation in magnetorheological elastomer and elastomer-ferromagnet composite driven by a magnetic field, Smart Materials and Structures, 13, pp. 309-316, 2004.

[12] Chen L., Gong X.L., Li W.H., Microstructures and viscoelastic properties of anisotropic magnetorheological elastomers, Smart Materials and Structures, 16, pp.2645-2650, 2007.

[13] Gong X.L., Chen L., Li J.F., Study of utilizable magnetorheological elastomers, International Journal of Modern Physics B, 21(28,29), pp. 4875-4882, 2007.

[14] Jiang W.G., Yao J.J., Gong X.L., Chen L., Enhancement in Magnetorheological Effect of Magnetorheological Elastomers by Surface Modification of Iron Particles, Chinese Journal of Chemical Physics, 21, pp. 87-92, 2008.

[15] Zhang X.Z., Gong X.L., Zhang P.Q, Li W.H., Existence of Bound-Rubber in Magnetorheological Elastomers and Its Influence on Material Properties, Chinese Journal of Chemical Physics, 20(2), pp. 173-179, 2007.

[16] Fan Y.C., Gong X.L., Jiang W.Q., Zhang W., Wei B., Effect of maleic anhydride on damping property of magnetorheological elastomers, Smart Materials and Structures, 19(5), pp. 55015-55022, 2010.

[17] Li J., Gong X., Zhu H., Jiang W., Influence of particle coating on dynamic mechanical behaviors of magnetorheological elastomers, Polymer Testing, 28, pp. 331-337, 2009.

[18] Boczkowska A., Awietjan S.F., Urethane Magnetorheological Elastomers Manufacturing, Microstructure and Properties, Solid State Phenomena, 154, pp.107-112, 2009.

[19] Boczkowska A., Awietjan S., Wejrzanowski T., Kurzydłowski K.J., Image analysis of the microstructure of magnetorheological elastomers, Journal of Materials Science, 44, pp.3135-3140, 2009. 